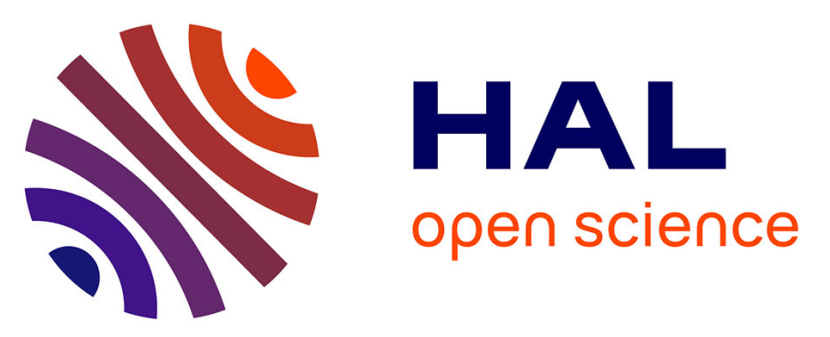

\title{
Electro-oxidized Epitaxial Graphene Channel Field-Effect Transistors with Single-Walled Carbon Nanotube Thin Film Gate Electrode
}

Palanisamy Ramesh, Mikhail E. Itkis, Elena Bekyarova, Feihu Wang, Sandip

Niyogi, Xiaoliu Chi, Claire Berger, Walt. A. de Heer, Robert C. Haddon

\section{To cite this version:}

Palanisamy Ramesh, Mikhail E. Itkis, Elena Bekyarova, Feihu Wang, Sandip Niyogi, et al.. Electrooxidized Epitaxial Graphene Channel Field-Effect Transistors with Single-Walled Carbon Nanotube Thin Film Gate Electrode. Journal of the American Chemical Society, 2010, 132, pp.14429-14436. 10.1021/ja101706j . hal-01002945

\section{HAL Id: hal-01002945 \\ https://hal.science/hal-01002945}

Submitted on 7 Jun 2014

HAL is a multi-disciplinary open access archive for the deposit and dissemination of scientific research documents, whether they are published or not. The documents may come from teaching and research institutions in France or abroad, or from public or private research centers.
L'archive ouverte pluridisciplinaire HAL, est destinée au dépôt et à la diffusion de documents scientifiques de niveau recherche, publiés ou non, émanant des établissements d'enseignement et de recherche français ou étrangers, des laboratoires publics ou privés. 


\title{
Electro-oxidized Epitaxial Graphene Channel Field-Effect Transistors with Single-Walled Carbon Nanotube Thin Film Gate Electrode
}

Palanisamy Ramesh, $\uparrow$ Mikhail E. Itkis, $\uparrow$ Elena Bekyarova, $\uparrow$ Feihu Wang,, , Sandip Niyogi, $\uparrow$ Xiaoliu Chi, $\uparrow, \S$ Claire Berger,,$\neq \neq$ Walt de Heer, $\neq$ and, Robert C. Haddon*, $†$

† Center for Nanoscale Science and Engineering, Departments of Chemistry, $\S$ Chemical \& Environmental Engineering, and Physics, University of California, Riverside, California 92521

| CNRS - Institut Néel, CNRS Grenoble, France

$\ddagger$ School of Physics, Georgia Institute of Technology, Atlanta, Georgia 30332

\begin{abstract}
We report the effect of electrochemical oxidation in nitric acid on the electronic properties of epitaxial graphene (EG) grown on silicon carbide substrates; we demonstrate the availability of an additional reaction channel in EG, which is not present in graphite but which facilitates the introduction of the reaction medium into the graphene galleries during electro-oxidation. The device performance of the chemically processed graphene was studied by patterning the EG wafers with two geometrically identical macroscopic channels; the electro-oxidized channel showed a logarithmic increase of resistance with decreasing temperature, which is ascribed to the scattering of charge carriers in a twodimensional electronic gas, rather than the presence of an energy gap at the Fermi level. Field-effect transistors were fabricated on the electro-oxidized and pristine graphene channels using single-walled carbon nanotube thin film top gate electrodes, thereby allowing the study of the effect of oxidative chemistry on the transistor performance of EG. The electro-oxidized channel showed higher values for the on-off ratio and the mobility of the graphene field-effect transistor, which we ascribe to the availability of high-quality internal graphene layers after electro-oxidation of the more defective top layers. Thus, the present oxidative process provides a clear contrast with previously demonstrated covalent chemistry in which sp3 hybridized carbon atoms are introduced into the graphitic transport layer of the lattice by carbon-carbon bond formation, thereby opening an energy gap.
\end{abstract}




\section{Introduction}

Graphene is a promising material for next generation electronic circuitry; current processes that are capable of producing large area graphene wafers include epitaxial growth from silicon carbide, ${ }^{1-4}$ chemical vapor deposition, ${ }^{5,6}$ and plasma assisted deposition. ${ }^{7,8}$ Such films possess electronic properties similar to those exhibited by microscopic single layer flakes obtained by exfoliation of graphite. ${ }^{9,10}$ The development of advanced electronic chips on the basis of large area graphene wafers can be approached by conventional physical lithographical techniques, but we have suggested the application of covalent chemistry to pattern graphene and to modify its electronic properties to fabricate a range of electronic devices, including wires, transistors, and dielectrics. ${ }^{11,12}$

Graphene consists of a honeycomb lattice of $\mathrm{sp}^{2}$ bonded carbon atoms, ${ }^{13}$ and the manipulation of the electronic properties by both ionic doping and covalent chemistry has been reported, ${ }^{11,12,14-20}$ in analogy with previous work on the fullerenes ${ }^{21,22}$ and carbon nanotubes. $^{23-27}$ The rich covalent chemistry available to carbon-based systems provides an opportunity to manipulate graphene well beyond that which is available in a typical electronics fabrication platform. In our previous work, we have reported the covalent modification of epitaxial graphene by spontaneous addition of nitrophenyl molecules. ${ }^{12}$ Here we demonstrate the progressive electro-oxidation of graphene layers and monitor the electronic properties as a function of the oxidative treatment. We find that electrooxidation carried out on the top surface leads to an improvement of the field-effect transistor performance of macroscopic EG channels, and we provide a model to explain this surprising result.

\section{Experimental Section}

We studied EG films grown on the $\mathrm{C}$-face of $\mathrm{SiC}$ substrates (Cree Inc., 4H SiC) with dimensions of $3.5 \times 4.5 \mathrm{~mm}^{2}{ }^{28,29}$ The as-prepared EG films were patterned with two identical macroscopic channels $\left(0.35 \times 1.2 \mathrm{~mm}^{2}\right)$ by following a stainless steel shadow mask using mechanical cutting with a precision knife with a titanium alloy blade. The electrical contacts to the channels were formed by e-beam evaporation of $10 \mathrm{~nm}$ Ti and $100 \mathrm{~nm}$ Au layers wired to the outside circuit via indium pads. The device isolation was tested by monitoring the resistance between the channels; the resulting channel configuration is given schematically in Figure 1a. The present patterning technique cannot be extended to the micro and nanoscale, but has the advantage of preserving the pristine state of the EG surface prior to chemical functionalization; it was recently found that lithography can leave residue on the surface of the graphene, which is difficult to completely eliminate. 30 In our investigation of alternative approaches, we utilized focused ion beam and laser cutting techniques for the channel patterning; in the channels, and we observed a significant distortion of the electrical properties of EG as well as visual damage of the surface due to the high energy irradiation. Thus, we chose mechanical cutting as the patterning technique that is best able to preserve the pristine state of the graphene surface before chemical functionalization. 
For the electro-oxidation experiment, a $1 \mathrm{~mm}$ diameter Teflon capillary tube mounted on an XYZ translational stage was used to position a drop of the electrolyte $\left(1 \mathrm{M} \mathrm{HNO}_{3}\right)$ on top of the test channel (channel T), while the reference channel (channel R) was maintained in its pristine state. The experimental configuration is shown schematically in Figure 1a; the capillary tube was connected to a peristaltic pump to precisely control the size of the electrolyte drop. The extension of the drop on the graphene surface was maintained at a diameter of $\sim 1.5 \mathrm{~mm}$, thereby covering most of the length of the narrow part of the active (electro-oxidized) channel T, and fully covering the width of this channel without spreading to the gold contacts or to the reference channel $\mathrm{R}$. The Pt wire mounted at the capillary tube and inserted into the electrolyte drop served as a counter/reference electrode, while the surface of the graphene channel was used as the working electrode in the cyclic voltammetry $(\mathrm{CV})$ experiments.

Atomic force microscope (AFM) imaging of the graphene channel was carried out using Dimension 5000 Veeco AFM equipment. Raman spectra were collected with a Nicolet Almega XR Raman microscope using $532 \mathrm{~nm}$ laser excitation. The maximum incident laser power on the sample was $0.5 \mathrm{~mW}$ with a $0.7 \mu \mathrm{m}$ laser spot size. The EG spectra usually contain features due to the $\mathrm{SiC}$ substrate; to extract the graphene spectrum, spectra of the underlying SiC wafer were collected by shifting the focus of the laser beam below the EG surface layer and then carrying out a subtraction.

\section{Results and Discussion}

The onset oxidation potential of graphene in nitric acid is apparent in the CV experiment (Figure $1 \mathrm{~b}$ ), and the peak in the oxidation current occurs at about $+0.6 \mathrm{~V}$, while the reverse scan does not show a wave due to a reduction process, indicating an irreversible chemical transformation. On the basis of the experimental CV (Figure 1b), we carried out potentiostatic oxidation by applying $+0.8 \mathrm{~V}$ to the working electrode (channel $\mathrm{T}$ ) against a $\mathrm{Pt}$ wire in $1 \mathrm{M} \mathrm{HNO}_{3}$ electrolyte confined over the active channel T. The oxidation potential was chosen to be more positive than the $\mathrm{CV}$ peak potential to avoid the diffusion limitation and to establish kinetic control over the process. To control the rate of the process and monitor the degree of oxidation of the EG, the current was limited to $50 \mathrm{nA}$ and the duration of the electro-oxidation steps was limited to 4-5 min. The typical time dependence of the electrochemical current during an electro-oxidation step is presented in Figure 1c; note that the first $100 \mathrm{~s}$ of the process corresponds to the current controlled regime (I e $50 \mathrm{nA}$ ), while the rest of the electrooxidation was carried out at a constant potential of $+0.8 \mathrm{~V}$. The current observed during electrolysis is comprised of polarization and charging of the EG (which accompanies intercalation) and oxidative processes (Figure 1c). During the initial oxidation step, the current is dominated by polarization and intercalation, but in the subsequent steps the contribution from intercalation is reduced and oxidation starts immediately $(<10 \mathrm{~s})$ after the application of the potential. After each oxidation step, the EG sample was washed with deionized water to remove residual acid and then rinsed with isopropanol and dried; the washing and drying steps were repeated several times until a stable resistance value was observed for the EG sample. Typically, 610 electro-oxidation steps were required to achieve a 2-fold increase of the channel resistance. We monitored the resistance of graphene channel $\mathrm{T}$ as the function of oxidation 
(Figure 1d); typically, a small decrease in resistance was observed for the first step of the electro-oxidation as a result of the charging (doping) and intercalation. ${ }^{31-34}$ With continued electro-oxidation, the resistance of the graphene channel increases nonlinearly with the electrochemical charge as the reaction penetrates additional layers.

Table 1 presents the resistance change and the corresponding electrochemical charge passed through the graphene channel. It shows that the EG samples can be progressively oxidized; the coulomb charge passed during the electrochemical oxidation steps correlates with the resistance change observed for the EG samples.

From the data in Table 1, we can estimate the degree of damage to the active area of the electro-oxidized channel. If we take the active area (A) of the experiment to be a circle of diameter $0.15 \mathrm{~cm}$, then we obtain an area, A ) $1.77 \times 10^{-2} \mathrm{~cm}^{2}$, and thus the number of carbon atoms in the active area per graphene layer is given by $\mathrm{N}_{\mathrm{C}}=\mathrm{C}_{\mathrm{C}} \times \mathrm{A}=6.73 \times 10^{13}$, where $C_{C}$ is the concentration of carbon atoms per unit area in graphite $\left(3.81 \times 10^{15} \mathrm{~cm}^{-2}\right)^{3.35}$ From Table 1, we can estimate the number of charges passed in the experiment (n), as $\mathrm{n}=$ $4.93 \times 10^{14}$ (EG-A), $1.23 \times 10^{15}$ (EG-B), and $2.89 \times 10^{14}$ (EGC). The overall stoichiometry of the reaction is uncertain but presumably involves between 1 and 4 charges per oxidized carbon atom; thus, the current passed is sufficient to react with 1.8-7.3 (EG-A), 4.6-18.3 (EG-B), and 1.1-4.3 (EG-C) graphene monolayers out of the original 4-8 layers on the starting EG wafer. Thus, it is clear that the electro-oxidation process has effectively reacted with a significant fraction of the graphene layers on the EG wafers.

It is well-known that the oxidation of graphite under acidic conditions is initiated by charging and intercalation, which is followed by the delamination of the lattice and the introduction of oxygen containing functional groups at the edges and into the graphene basal planes. ${ }^{31-34}$ Electrochemical oxidation has also been used to introduce defect sites into individual carbon nanotubes ${ }^{27}$ and recently a cooperative mechanism of oxygen attachment to the carbon nanotube walls was proposed. ${ }^{36,37}$ In the case of few-layer graphene (FLG), electro-oxidation is the most suitable technique for the controlled etching and introduc- tion of chemical functionalities, as opposed to the chemical oxidation methods used for the bulk preparation of graphite oxide. ${ }^{38,39}$ The observed irreversible oxidation implies that oxygen functional groups are attached to graphene through a covalent bond. Electrochemical oxidation in nitric acid of bulk graphite in various forms such as HOPG and glassy carbon is well documented in the literature, ${ }^{31-34}$ and the irreversible oxidation observed for graphene is in accordance with these results. Spectroscopic studies suggest the presence of functional groups such as phenols, esters, ethers, epoxides, and carboxylates, as the covalently bonded species that are introduced into graphite as a result of the oxidation. ${ }^{35,38,40-43}$

We conducted AFM and Raman studies of EG at different stages of the electro-oxidation to understand the nanoscale aspects of the chemistry. An AFM image of the pristine sample in Figure 2a shows the atomically smooth EG surface overlaid with a pattern of ridges, which is typical for EG grown on SiC substrates. ${ }^{44-46}$ The occurrence of ridges in EG is attributed to the compressive stress that accompanies the cooling of the wafer after the growth of EG at elevated temperatures as a result of the difference in the thermal 
coefficient of expansion of sample and substrate; thus, the ridges occur because of thermally induced buckling of the FLG due to the stress induced by cooling. ${ }^{44-46}$ The ridges (pleats, folds) are of larger dimensions (height, $\mathrm{h} \approx 10 \mathrm{~nm}$ ) than the features that arise where the FLG surface crosses a step edge, where the height is typically $\mathrm{h} \approx 1 \mathrm{~nm}$. As we show below, the ridges in EG introduce an additional reaction channel that is not present in other flat forms of $\mathrm{sp}^{2}$ carbon. Because the ridges introduce local curvature into the graphene sheets, they are expected to provide sites of enhanced reactivity based on previous work on the chemistry of the fullerenes 22 and carbon nanotubes 26 and the known relationship between curvature, pyramidalization, and reactivity of formally $\mathrm{sp}^{2}$ hybridized carbon. ${ }^{22,26,47}$

As the electro-oxidation progresses beyond the initial charging and edge intercalation processes, sp3 functionalities containing carbon-oxygen bonds are introduced into the graphene surface as point defects that are responsible for the increase in the surface roughness (Figure 2b). As the electro-oxidation continues, we observed the initiation of line defects (Figure 2c), which propagate along the ridges due to their enhanced reactivity, and we suggest that the formation of these well defined line-defects facilitates electrolyte access to the underlying EG galleries (Figure 2d), and this provides an additional reaction channel for EG that is not present in other flat carbons such as graphite. During further oxidation, we observed a partial delamination of the graphene layers along these line defects (Figure 2e), indicating the interlayer electrolyte intercalation, which partially lifts off the EG layers and provides further access to the underlying layers. We did not observe a uniform layer by layer delamination, lift-off, or etching, rather a diffusion like propagation of the reaction into the deeper layers. We also observed in some cases an essentially complete delamination with exposure of the $\mathrm{SiC}$ substrate (Figure 2f).

We conducted a sequential Raman study of sample EG-D in (a) pristine state, and after (b) 10 and (c) 20 min of electro-oxidation. To follow the consecutive changes of EG during the electrochemical oxidation, we collected data in a grid pattern with 5 or $10 \mu \mathrm{m}$ steps across the $100 \times 400 \mu \mathrm{m}$ area along the EG test channel. The resulting Raman spectra for each oxidation step were averaged and are presented in Figure 3a for different stages of the oxidation; the averaging is required to produce a statistical analysis of the chemistry occurring over the area of the reaction channel. ${ }^{48}$ The pristine EG sample shows the prominent $G$ and 2D-band features at frequencies 1581 and $2689 \mathrm{~cm}^{-1}$, respectively, which manifest the presence of graphene layers on the $\mathrm{SiC}$ surface. With increasing oxidation time, the intensities of $\mathrm{G}$ and $2 \mathrm{D}$ bands decrease, and the $\mathrm{D}$ band at $1340 \mathrm{~cm}^{-1}$, which is known to be activated by the presence of $\mathrm{sp} 3$ carbon centers in the conjugated sp2 network, is developed. The resulting increase of the ratio of intensities of $D$ to $G$ bands (ID/IG) with electro-oxidation time (Figure 3b) correlates with increasing concentration of the point and line defects observed in AFM images in Figure 2 and the increasing roughness of the oxidized EG surface. The decreasing intensity of $2 \mathrm{D}$ bands with electrooxidation time (Figure $3 \mathrm{~b}$ ) qualitatively indicates the thinning of EG layers and correlates with the increase of the channel resistance (Figure 1d), although a quantitative relationship between the intensity of 2D Raman mode and the number of layers of EG has yet to be established. 
Figure 4 presents the temperature dependence of the resistance of the two EG channels before and after electro-oxidation of sample EG-A. In the initial pristine state, both channels show a similar metallic temperature dependence of the resistance in the temperature range 360-200 K. At low temperatures, we observed some differences: Channel $\mathrm{R}$ shows $\sim 10 \%$ increase of resistance with decreasing temperature from 200 to 4.2 K, while channel T preserves its metallic behavior (decrease of the resistance with decreasing temperature). Variations in the transport behavior of macroscopic pristine EG samples have been observed previously and are due to a variety of factors including the inhomogeneity in EG growth over the macroscopic SiC wafers that influence the density of point defects and the concentration of charge carriers and slight variations in the transfer integrals at the ridges (as discussed above), due to misalignment of the $\pi$ orbitals, ${ }^{26}$ which taken together influence the scattering rate, as demonstrated by Hall measurements on large area EG wafers. ${ }^{49}$

After electro-oxidation, we observed an approximately 2-fold increase of the room temperature resistance of the oxidized channel $\mathrm{T}$, and the temperature dependence of the resistance changed drastically from metallic to nonmetallic over the whole temperature range with a total increase of resistance of $\sim 80 \%$ from 360 to $4.2 \mathrm{~K}$. The resistance of channel $\mathrm{R}$ and its temperature dependence remained essentially unchanged despite the fact that this channel was subjected to the solvents utilized during the washing and rinsing cycles that were applied to channel $\mathrm{T}$ after the oxidation steps.

To test if the nonmetallic behavior observed in the chemically processed channel is associated with the opening of an energy gap as a result of electro-oxidation, we present the temperature dependence of resistance data $\mathbf{R}(\mathbf{T})$ in the form of an Arrhenius plot in Figure 5a. Clearly this analysis does not lead to the linear relationship that is expected for an intrinsic semiconductor with a well-defined energy gap, and the plot shows a variable slope with a temperature-dependent activation energy, which changes from $1.9 \mathrm{meV}$ in the vicinity of room temperature to $0.03 \mathrm{meV}$ at helium temperatures, which is less than the room temperature thermal energy $[\mathbf{k B T}=26 \mathrm{meV}(\mathbf{T}=300 \mathrm{~K})]$. Moreover, there is a striking similarity in the shape of the resistance curves, $\mathbf{R}(\mathbf{T})$, of the oxidized and pristine channels as well as similar values for the activation energies in the corresponding temperature ranges. The nature of the observed $\mathbf{R}(\mathbf{T})$ dependence becomes clear when the resistance is plotted as a function of the logarithm of the temperature (Figure $5 \mathrm{~b}$ ); the function $\mathbf{R}=\mathbf{R}_{0}+\mathbf{A} * \ln \left(\mathbf{T} / \mathbf{T}_{0}\right)$ satisfactorily fits the experimental data over the temperature range from 50 to $5 \mathrm{~K}$. This type of logarithmic temperature dependence was observed in a number of two dimensional electron gas (2DEG) systems first at low temperatures $<1 \mathrm{~K}$ in thin metal (AuPd) films ${ }^{50}$ and silicon inversion Layers ${ }^{51}$ and later up to temperatures of $100 \mathrm{~K}$ in ultrathin Pt films ${ }^{52}$ and indium oxide films. ${ }^{53}$

Theoretically this logarithmic temperature dependence was ascribed to weak localization 54-56 or electron-electron interactions ${ }^{57,58}$ in 2DEG systems with disorder and discussed in a number of reviews. ${ }^{59,60}$ Thus, the quasi-linear $\mathrm{R}$ versus $\ln \mathrm{T}$ dependence presented in Figure $5 \mathrm{~b}$ indicates that the nonmetallic character of the electrical conductivity originates from scattering in a 2D electronic system with some degree of disorder, rather than from the opening of an energy gap at the Fermi level corresponding to an oxidation-induced 
metal-insulator transition. We attribute the increase in film disorder in the electro-oxidized channel $\mathrm{T}$ to the contribution of additional scattering centers that are introduced by chemical functionalization. Despite the change in resistance, the logarithmic character of the temperature dependence after electro-oxidation remains unchanged, which indicates that the $2 \mathrm{D}$ nature of charge carrier scattering is preserved. The oxidation process reported here contrasts with previously demonstrated functionalization reactions of carbon nanotubes ${ }^{23-26}$ and graphene, ${ }^{11,12}$ where the formation of covalent carbon-carbon bonds changes the hybridization of the graphitic carbon atoms from $\mathrm{sp}^{2}$ to $\mathrm{sp}^{3}$ and serves to introduce a well-defined energy gap.

Figure 6a presents the schematic configuration of the field effect transistors prepared on the basis of two parallel EG channels, and Figure 6b shows an optical micrograph of the device. To fabricate the dielectric, we coated the graphene channel with a parylene film by vacuum deposition; ${ }^{61,62}$ briefly, the parylene was sublimed at $120^{\circ} \mathrm{C}$ at the end of the furnace, cleaved by passing through the pyrolysis zone, which was kept at $650{ }^{\circ} \mathrm{C}$, and polymerized on the epitaxial graphene sample placed in the room temperature region of the reactor tube, which was maintained at a pressure of 0.1 Torr. The thickness of the dielectric layer was $\sim 1 \mu \mathrm{m}$ as measured by a Dectak profilometer. For the large area macroscopic FETs utilized in the current study, there is a much higher probability of device failure due to pinholes in the gate dielectric in comparison with micrometer size devices. To increase the yield of functional FETs, we utilized a conducting SWNT thin film as the top gate electrode; the $40 \mathrm{~nm}$ thick semitransparent SWNT film was prepared by vacuum filtration ${ }^{63,64}$ and placed on top of the dielectric layer so that it spanned both graphene channels. The SWNT film functions as an integrated two-dimensional conducting network with in-plane alignment, which suppresses the formation of pin-holes due to the high aspect ratio of the carbon nanotubes, and is much superior to the vapor deposition of metals such as gold for the present application. The graphene devices were annealed in vacuum at $10-{ }^{6}$ Torr for $12 \mathrm{~h}$ at $100{ }^{\circ} \mathrm{C}$ before deposition of the gate dielectric and after the completion of the FET fabrication.

The transfer characteristics of the pristine and oxidized graphene FET channels were measured simultaneously using custom LabVIEW software. The source drain current Isd was measured at a voltage Vsd ) $100 \mathrm{mV}$ as a function of gate voltage $\mathrm{Vg}$ in the temperature range from 300 to $4.4 \mathrm{~K}$, and the results for sample EG-A are presented in Figure $6 \mathrm{c}$ and $\mathrm{d}$. The temperature dependence of the channel resistance and the resistance values at zero gate voltage correlate with the results presented in Figure 4 before deposition of parylene showing that the transport characteristics of EG are not affected by the parylene coating in accord with recently published observations on exfoliated graphene.65 The pristine channel R (Figure 6c) showed p-type behavior, the origin of which is not fully understood,49 but may depend on the EG growth conditions, defects and impurities in the underlying $\mathrm{SiC}$ wafer, and environmental doping. The oxidized channel $\mathrm{T}$ showed a significantly increased degree of $\mathrm{p}$-doping due to the electrooxidation with the neutrality point shifted to positive polarity beyond the maximum gate voltage that could be applied $(110 \mathrm{~V})$. The most drastic difference between devices was observed in the on-off ratio of the FET devices: 1.9 for the oxidized channel $\mathrm{T}$ and 1.03 (3\% increase of the current) for the pristine channel $\mathrm{R}$ at $\mathrm{T}=4.4 \mathrm{~K}$, showing the enhancement of the 
FET performance of the EG channel after electro-oxidation with nitric acid. Similar improvements of the on-off ratio after electro-oxidation were observed in the two other EG devices that were characterized, and the results for the EG-C sample are presented in Figure $6 \mathrm{e}$ (channel R) and Figure $6 \mathrm{f}$ (channel T), demonstrating the reproducibility of the effect.

From the slope of source-drain current versus gate voltage $\mathrm{dII}_{\mathrm{d}} / \mathrm{dV}_{\mathrm{g}}$ in the negative $\mathrm{V}_{\mathrm{g}}$ range, we calculated the carrier drift mobility $\mu=\left(\left(\mathrm{L} \cdot \mathrm{t}_{\mathrm{PAR}}\right) /\left(\varepsilon_{\mathrm{PAR}} \cdot \varepsilon 0 \cdot \mathrm{V}_{\mathrm{sd}} \cdot \mathrm{W}\right)\right) \cdot \mathrm{dIs}_{\mathrm{d}} / \mathrm{dV}_{\mathrm{g}}$, utilizing values of length $\mathrm{L}=1.3 \mathrm{~mm}$, width $\mathrm{W}=0.35 \mathrm{~mm}$ for the FET channel, thickness $\mathrm{t}_{\mathrm{PAR}}=1 \mu \mathrm{m}$, dielectric constant $\varepsilon_{\mathrm{PAR}}=2.65$ for the parylene dielectric layer, where $\varepsilon_{0}$ is the vacuum dielectric constant and $\mathrm{Vsd}=0.1 \mathrm{~V}$ is the source-drain voltage. The mobility data for both FET channels as a function of temperature are presented in Figure 7, and it may be seen that the drift mobility in the pristine channel is significantly lower than in the oxidized channel. For the oxidized channel, the mobility increases as the temperature is lowered and reaches a maximum value of $\mu=450$ and $370 \mathrm{~cm}^{2} / \mathrm{V} \cdot \mathrm{s}$ for samples EG-C and EG-A, respectively, followed by a drop below $25 \mathrm{~K}$, which is typical of a transition from phonon to impurity scattering.

The maximum values of the mobility for the pristine channels are $\mu$ ) 170 and $70 \mathrm{~cm}_{2} / \mathrm{V} \cdot \mathrm{s}$ for samples EG-C and EG-A, respectively, which are at the low end of the published data for $\mathrm{EG}^{48,49}$ and much lower than the best results. ${ }^{1-3,48}$ The low values of mobility reported herein may originate from variations in the sample quality, 49 but it is probably due to the macroscopic size of our devices, which are not directly comparable to the lithographically defined, microscopic FETs that are typically confined to the highest quality areas of EG.

The observed increase of resistance together with the improved on-off ratio in the EG channel after electro-oxidation is consistent with a picture in which the top 3-4 layers of the initial 6-8 layers are excluded from the electrical transport by a combination of point and line defects, delamination, and oxidative removal, while the underlying layers that are responsible for the charge transport remain relatively defect free. The gate voltage couples most strongly to the layers adjacent to the gate dielectric and is screened from the deeper layers, so the decrease in the total number of active layers will improve the utilization of the lower layers in the FLG stack. The increase of mobility after nitric acid electrooxidation was not expected and is more difficult to comprehend at first sight, but can be explained by assuming that the uppermost layers that are partially removed or excluded from the conductive pathway by the oxidation are the most defective as a result of the bottom up growth of EG. ${ }^{3,66,67}$ Thus, by excluding these defective layers from the electrical transport, the electro-oxidation process activates high-quality lower EG layers, thereby improving not only the on-off ratio, but also the effective drift mobility and enhancing all aspects of the FET performance.

In summary, we conducted a controlled electrochemical oxidation of macroscopic multilayer epitaxial graphene (EG) channels under potentiostatic conditions, and we showed the presence of an additional reaction channel in EG that is not present in graphite. The electro-oxidized channel showed a logarithmic increase of resistance with decreasing temperature, which can be ascribed to the scattering of the charge carriers in a 
two-dimensional electron gas rather than to the presence of an energy gap at the Fermi level. We observed an improved field-effect transistor performance in the electro-oxidized channel, which can be ascribed to the gating of high-quality internal EG layers after electro-oxidative removal of the more defective outside layers. More efficient chemistries that are capable of opening an energy gap through carbon-carbon bond formation to the basal plane of graphene ${ }^{11,12}$ further enhance the prospects for the development of graphene-based electronics.

\section{Acknowledgment.}

We acknowledge financial support from DOD/DMEA under contract H94003-10-2-1003 and NSF-MRSEC through contract DMR-0820382.

\section{References}

(1) Berger, C.; Song, Z.; Li, T.; Li, X.; Ogbazghi, A. Y.; Feng, R.; Dai,Z.; Marchenkov, A. N.; Conrad, E. H.; First, P. N.; de Heer, W. A. J.Phys. Chem. B 2004, 108, 1991219916.

(2) Berger, C.; Song, Z. M.; Li, X. B.; Wu, X. S.; Brown, N.; Naud, C.;Mayou, D.; Li, T. B.; Hass, J.; Marchenkov, A. N.; Conrad, E. H.;First, P. N.; de Heer, W. A. Science 2006, 312, 1191-1196.

(3) Emtsev, K. V.; Bostwick, A.; Horn, K.; Jobst, J.; Kellogg, G. L.; Ley, L.; McChesney, J. L.; Ohta, T.; Reshanov, S. A.; Rohrl, J.; Rotenberg,E.; Schmid, A. K.; Waldmann, D.; Weber, H. B.; Seyller, T. Nat.Mater. 2009, 8, 203-207.

(4) Lin, Y. M.; Dimitrakopoulos, C.; Jenkins, K. A.; Farmer, D. B.; Chiu,H. Y.; Grill, A.; Avouris, P. Science 2010, 327, 662-662.

(5) Kim, K. S.; Zhao, Y.; Jang, H.; Lee, S. Y.; Kim, J. M.; Kim, K. S.;Ahn, J. H.; Kim, P.; Choi, J. Y.; Hong, B. H. Nature 2009, 457, 706-710.

(6) Li, X. S.; Cai, W. W.; An, J. H.; Kim, S.; Nah, J.; Yang, D. X.; Piner,R.; Velamakanni, A.; Jung, I.; Tutuc, E.; Banerjee, S. K.; Colombo,L.; Ruoff, R. S. Science 2009, 324, 1312-1314.

(7) Malesevic, A.; Vitchev, R.; Schouteden, K.; Volodin, A.; Zhang, L.;Van Tendeloo, G.; Vanhulsel, A.; Van Haesendonck, C. Nanotechnology2008, 19, 305604.

(8) Yuan, G. D.; Zhang, W. J.; Yang, Y.; Tang, Y. B.; Li, Y. Q.; Wang,J. X.; Meng, X. M.; He, Z. B.; Wu, C. M. L.; Bello, I.; Lee, C. S.;Lee, S. T. Chem. Phys. Lett. 2009, 467, 361-364.

(9) Novoselov, K. S.; Geim, A. K.; Morozov, S. V.; Jiang, D.; Zhang,Y.; Dubonos, S. V.; Grigorieva, I. V.; Firsov, A. A. Science 2004,306, 666-669.

(10) Novoselov, K. S.; Jiang, D.; Schedin, F.; Booth, T. J.; Khotkevich,V. V.; Morozov, S. V.; Geim, A. K. Proc. Natl. Acad. Sci. U.S.A.2005, 102, 10451-10453.

(11) Bekyarova, E.; Itkis, M. E.; Ramesh, P.; Haddon, R. C. Phys. StatusSolidi RRL 2009, 3, 184-186.

(12) Bekyarova, E.; Itkis, M. E.; Ramesh, P.; Berger, C.; Sprinkle, M.; deHeer, W. A.; Haddon, R. C. J. Am. Chem. Soc. 2009, 131, 1336-1337. 
(13) Dresselhaus, M. S.; Dresselhaus, G.; Eklund, P. C. Science of Fullerenes and Carbon Nanotubes; Academic: San Diego, CA, 1996.

(14) Das, A.; Pisana, S.; Chakraborty, B.; Piscanec, S.; Saha, S. K.; Waghmare, U. V.; Novoselov, K. S.; Krishnamurthy, H. R.; Geim, A. K.; Ferrari, A. C.; Sood, A. K. Nat. Nanotechnol. 2008, 3, 210- 215.

(15) Chen, W.; Chen, S.; Qi, D. C.; Gao, X. Y.; Wee, A. T. S. J. Am. Chem. Soc. 2007, $129,10418-10422$.

(16) Wu, X. S.; Sprinkle, M.; Li, X. B.; Ming, F.; Berger, C.; de Heer, W. A. Phys. Rev. Lett. 2008, 101, 026801.

(17) Farmer, D. B.; Golizadeh-Mojarad, R.; Perebeinos, V.; Lin, Y.-M.; Tulevski, G. S.; Tsang, J. C.; Avouris, P. Nano Lett. 2009, 9, 388- 392.

(18) Elias, D. C.; Nair, R. R.; Mohiuddin, T. M. G.; Morozov, S. V. B., P.; Halsall, M. P.; Ferrari, A. C.; Boukhvalov, D. W.; Katsnelson, M. I.; Geim, A. K.; Novoselov, K. S. Science 2009, 323, 610-613.

(19) Ryu, S.; Han, M. Y.; Maultzsch, J.; Heinz, T. F.; Kim, P.; Steigerwald, M.; Brus, L. E. Nano Lett. 2008, 8, 4597-4602.

(20) Jung, N.; Kim, N.; Jockusch, S.; Turro, N. J.; Kim, P.; Brus, L. Nano Lett. 2009, 9, 4133-4137.

(21) Haddon, R. C. Acc. Chem. Res. 1992, 25, 127-133.

(22) Haddon, R. C. Science 1993, 261, 1545-1550.

(23) Chen, J.; Hamon, M. A.; Hu, H.; Chen, Y.; Rao, A. M.; Eklund, P. C.; Haddon, R. C. Science 1998, 282, 95-98.

(24) Hu, H.; Zhao, B.; Hamon, M. A.; Kamaras, K.; Itkis, M. E.; Haddon, R. C. J. Am. Chem. Soc. 2003, 125, 14893-14900.

(25) Kamaras, K.; Itkis, M. E.; Hu, H.; Zhao, B.; Haddon, R. C. Science 2003, 301, 1501.

(26) Niyogi, S.; Hamon, M. A.; Hu, H.; Zhao, B.; Bhowmik, P.; Sen, R.; Itkis, M. E.; Haddon, R. C. Acc. Chem. Res. 2002, 35, 1105-1113.

(27) Goldsmith, B. R.; Coroneus, J. G.; Khalap, V. R.; Kane, A. A.; Weiss, G. A.; Collins, P. G. Science 2007, 315, 77-81.

(28) de Heer, W. A.; Berger, C.; Wu, X. S.; First, P. N.; Conrad, E. H.; Li, X. B.; Li, T. B.; Sprinkle, M.; Hass, J.; Sadowski, M. L.; Potemski, M.; Martinez, G. Solid State Commun. 2007, 143, 92-100.

(29) Rolling, E.; Gweon, G. H.; Zhou, S. Y.; Mun, B. S.; McChesney, J. L.; Hussain, B. S.; Fedorov, A.; First, P. N.; de Heer, W. A.; Lanzara, A. J. Phys. Chem. Solids 2006, 67, 2172-2177.

(30) Ishigami, M.; Chen, J. H.; Cullen, W. G.; Fuhrer, M. S.; Williams, E. D. Nano Lett. 2007, 7, 1643-1648.

(31) Choo, H. S.; Kinumoto, T.; Jeong, S. K.; Iriyama, Y.; Abe, T.; Ogumi, Z. J. Electrochem. Soc. 2007, 154, B1017-B1023.

(32) Hathcock, K. W.; Brumfield, J. C.; Goss, C. A.; Irene, E. A.; Murray, R. W. Anal. Chem. 1995, 67, 2201-2206.

(33) Kepley, L. J.; Bard, A. J. Anal. Chem. 1988, 60, 1459-1467.

(34) Bowling, R.; Packard, R. T.; Mccreery, R. L. Langmuir 1989, 5, 683- 688.

(35) Niyogi, S.; Bekyarova, E.; Itkis, M. E.; McWilliams, J. L.; Hamon, M. A.; Haddon, R. C. J. Am. Chem. Soc. 2006, 128, 7720-7721. 
(36) Ashraf, M. K.; Bruque, N. A.; Pandey, R. R.; Collins, P. G.; Lake, R. K. Phys. Rev. B 2009, 79, 115428.

(37) Kanai, Y.; Khalap, V. R.; Collins, P. G.; Grossman, J. C. Phys. Rev. Lett. 2010, 104, 066401

\section{Figure Captions}

Figure 1. (a) Schematic illustration of electrochemical oxidation of epitaxial graphene, where channel $\mathrm{T}$ is the oxidized graphene and channel $\mathrm{R}$ is the reference pristine graphene; and (b) cyclic voltammogram of epitaxial graphene electrode in 1 M HNO3. (c) Potentiostatic electro-oxidation of epitaxial graphene. (d) Resistance of graphene channel $\mathrm{T}$ as a function of electrochemical charge.

Figure 2. AFM images of EG-C sample. (a) AFM of the pristine channel R (roughness rms ) 0.30 ( 0.09). (b-e) Transformation of EG surface during the oxidation steps: (b) rough surface due to point defects; (c) example of the propagation of a line defect; (d) well-defined line for the electrolyte access; and (e) partial delamination of the top graphene layers (rms ) 0.52 (0.06). (f) Delamination along ridges showing the folded EG layer exposing the bare $\mathrm{SiC}$ substrate.

Figure 3. (a) Raman spectra of EG-D pristine sample (red line) and after 10 min (blue line) and 20 min (black line) electro-oxidation; (b) intensity of 2D-band (red 9, left scale) and D-band to G-band intensities ratio (blue 2, right scale) as a function of electro-oxidation time.

Figure 4. Temperature dependences of resistance of graphene channels before and after oxidation. The resistance data were taken before FET fabrication without gating the channels.

Figure 5. Temperature dependences of resistances of pristine and electro-oxidized graphene channels presented as (a) Arrhenius and (b) semilogarithmic plot.

Figure 6. (a) Schematics and (b) microscope image of SiC substrate with two FETs based on pristine and electro-oxidized graphene channels with SWNT thin film gate electrode. Dependence of the channel current on gate voltage for two EG samples: EG-A, pristine (c) and electro-oxidized graphene (d); EG-C, pristine (e) and electrooxidized (f) graphene channels at $5 \mathrm{~K}$.

Figure 7. FET mobility as a function of temperature in pristine and electro-oxidized channels for two different EG samples: EG-A, open symbols; and EG-C, closed symbols. 


\section{Table and Figures}

Table 1. Resistance Change and Electrochemical Charge

\begin{tabular}{cccccc} 
wafer & $\begin{array}{c}\text { initial } \\
\text { resistance } \\
\text { (ohm) }\end{array}$ & $\begin{array}{c}\text { final } \\
\text { resistance } \\
\text { (ohm) }\end{array}$ & $\begin{array}{c}\text { resistance } \\
\text { change } \\
(\%)\end{array}$ & $\begin{array}{c}\text { electrochemical } \\
\text { charge } \\
\text { (coulomb) }\end{array}$ & $\begin{array}{c}\text { equivalents } \\
\text { (mol) }\end{array}$ \\
\hline EG-A & 5740 & 11600 & $102 \%$ & $7.90 \times 10^{-5}$ & $8.19 \times 10^{-10}$ \\
EG-B & 6500 & 22300 & $243 \%$ & $1.98 \times 10^{-4}$ & $2.05 \times 10^{-9}$ \\
EG-C & 5000 & 7850 & $57 \%$ & $4.64 \times 10^{-5}$ & $4.81 \times 10^{-10}$ \\
\hline
\end{tabular}


(a)

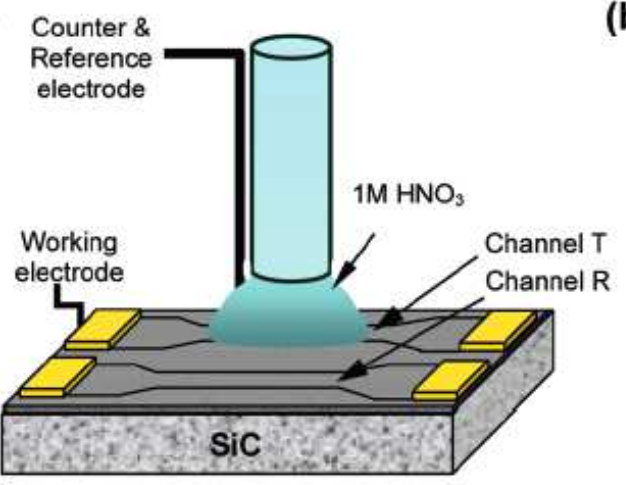

(c)

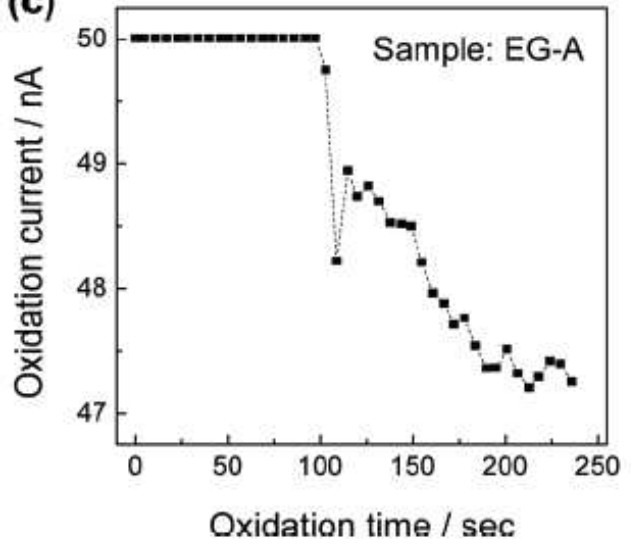

(b)

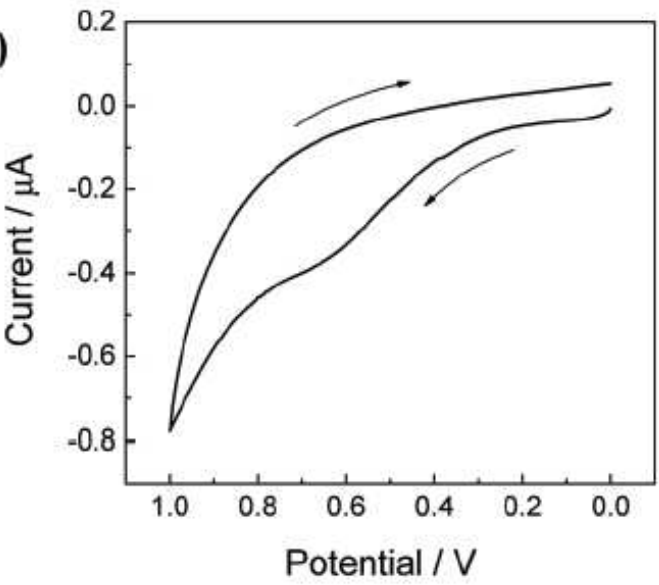

(d)

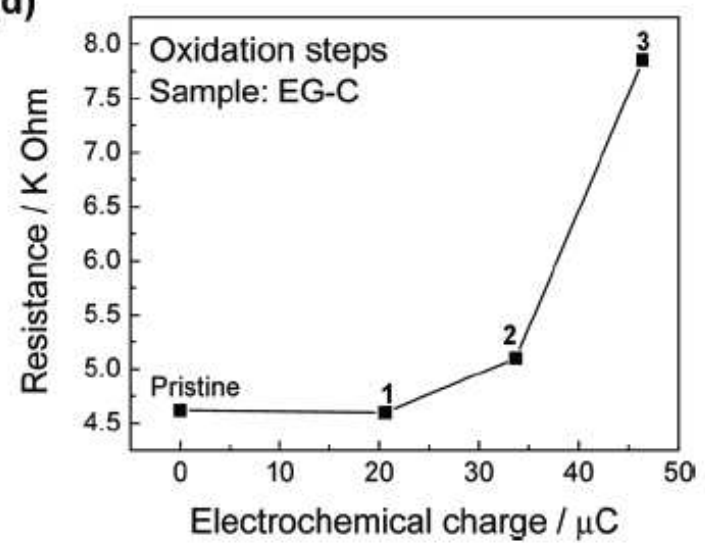

Figure 1 

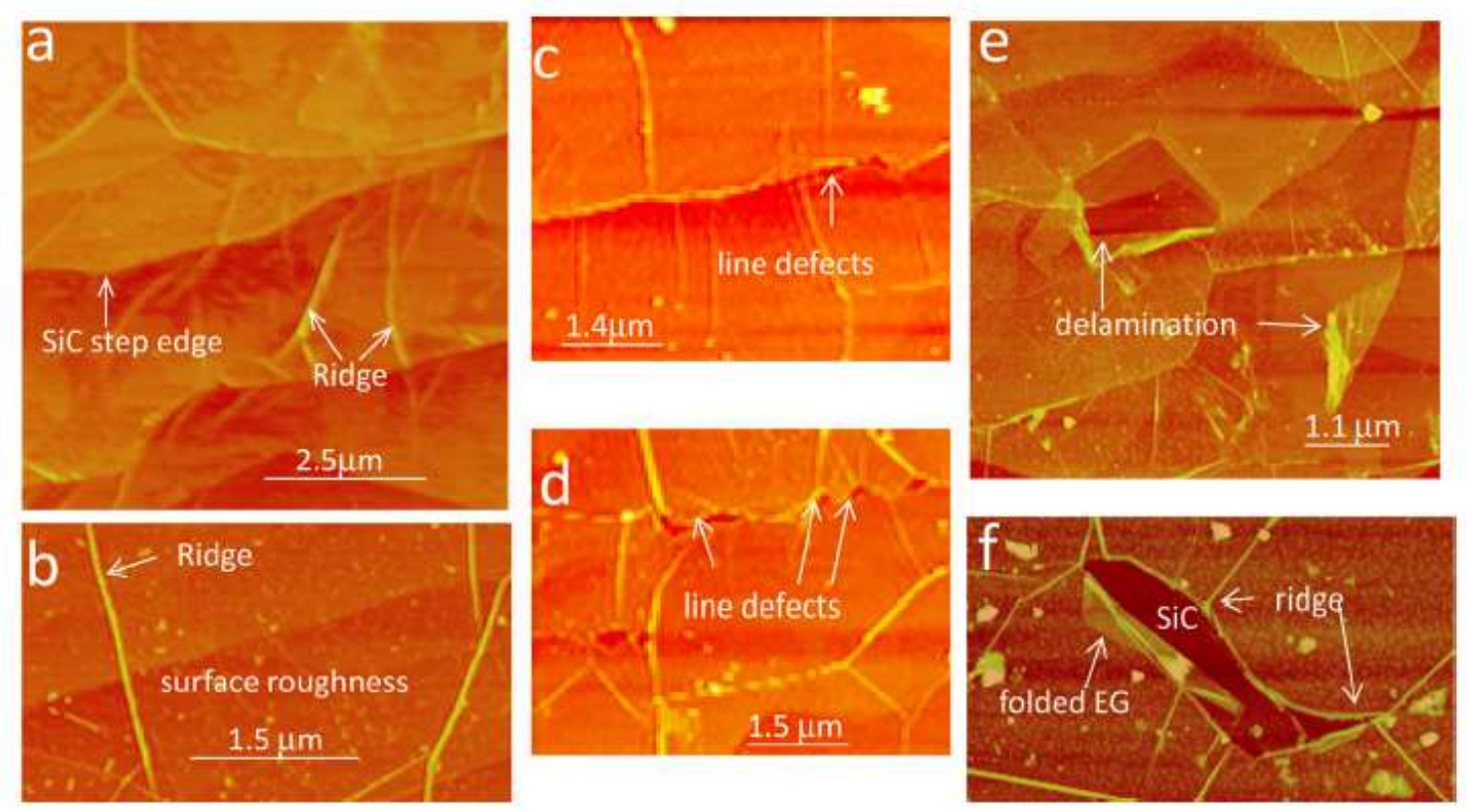

Figure 2 

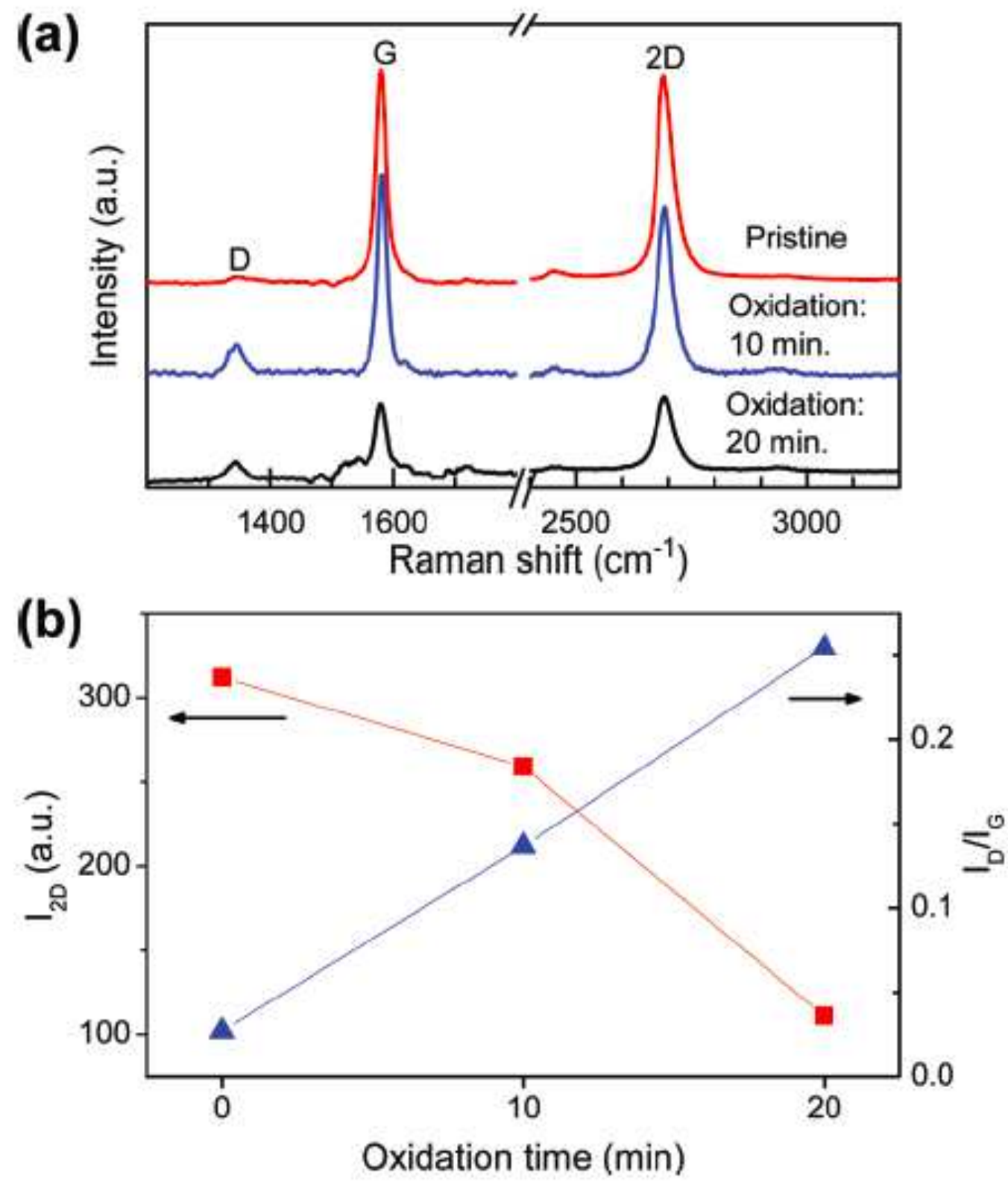

Figure 3 


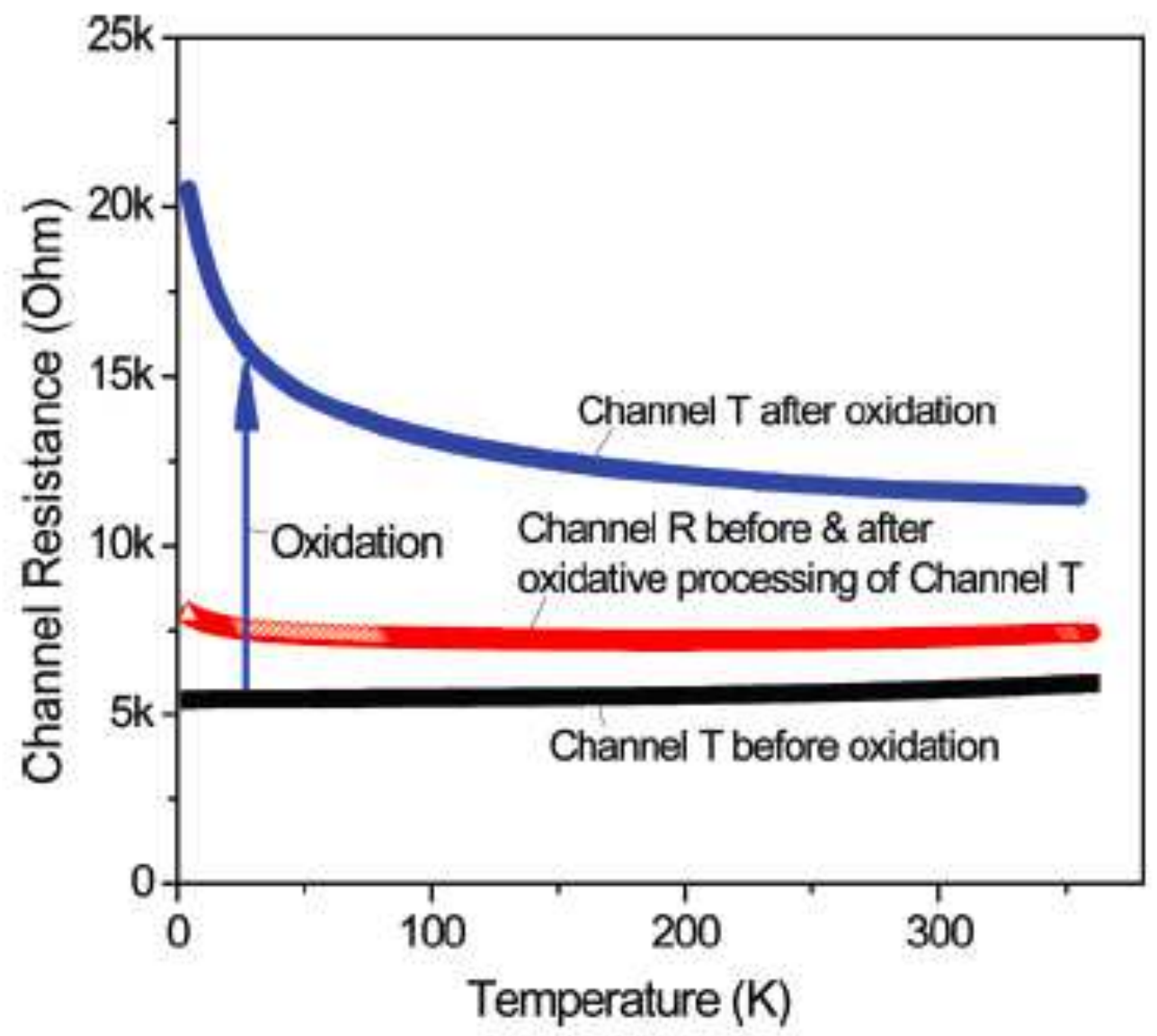

Figure 4 

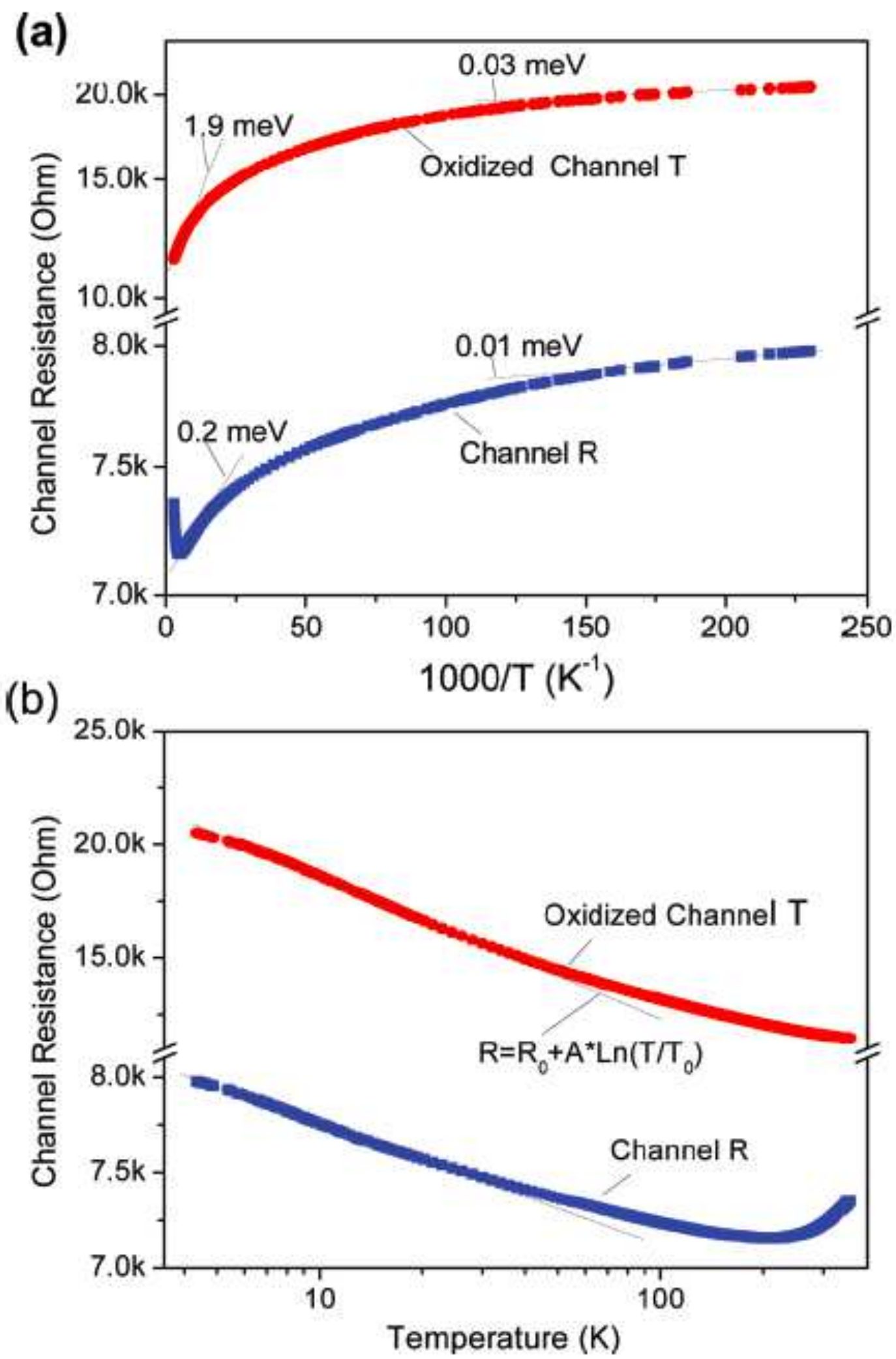

Figure 5 

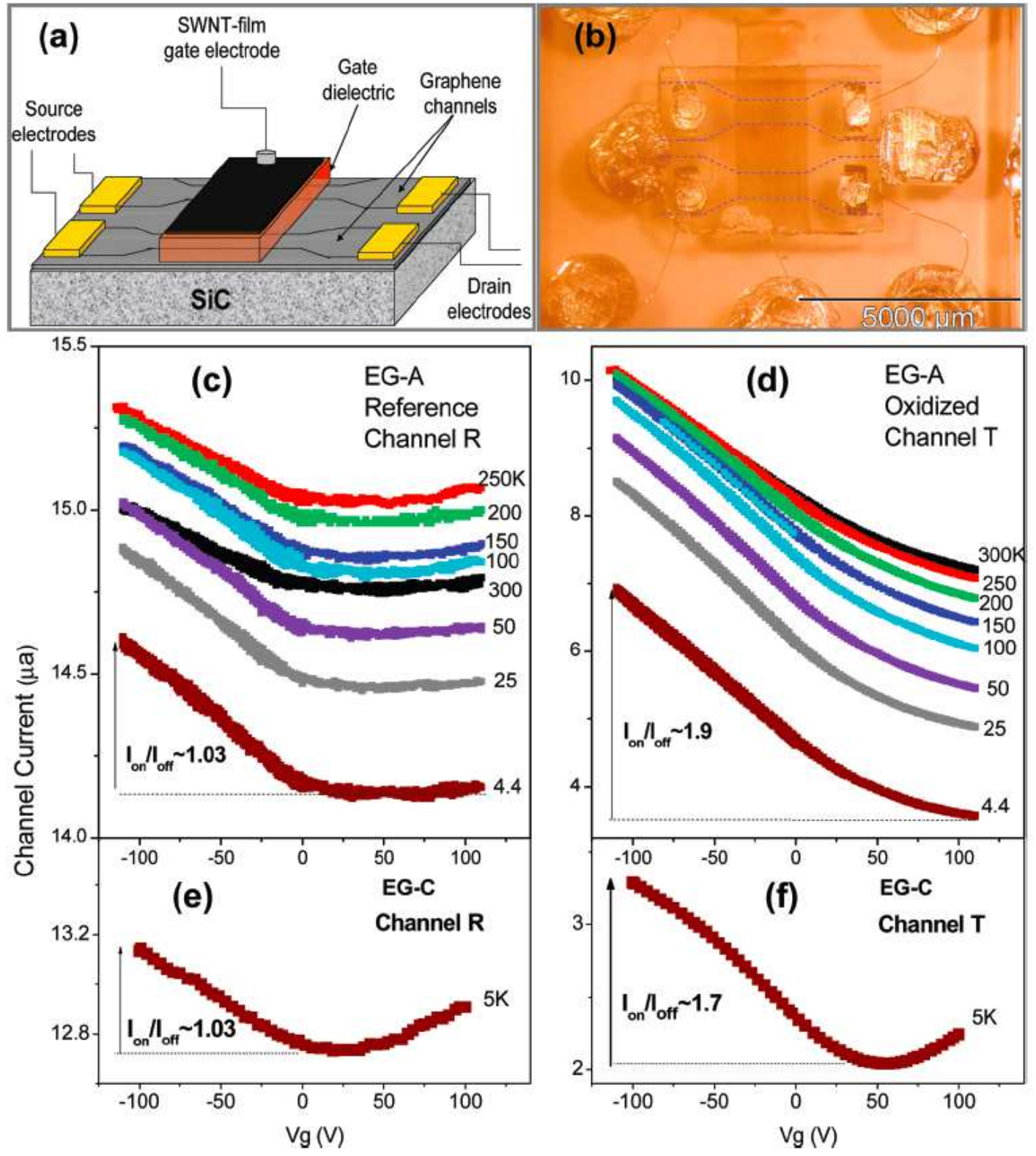

Figure 6 


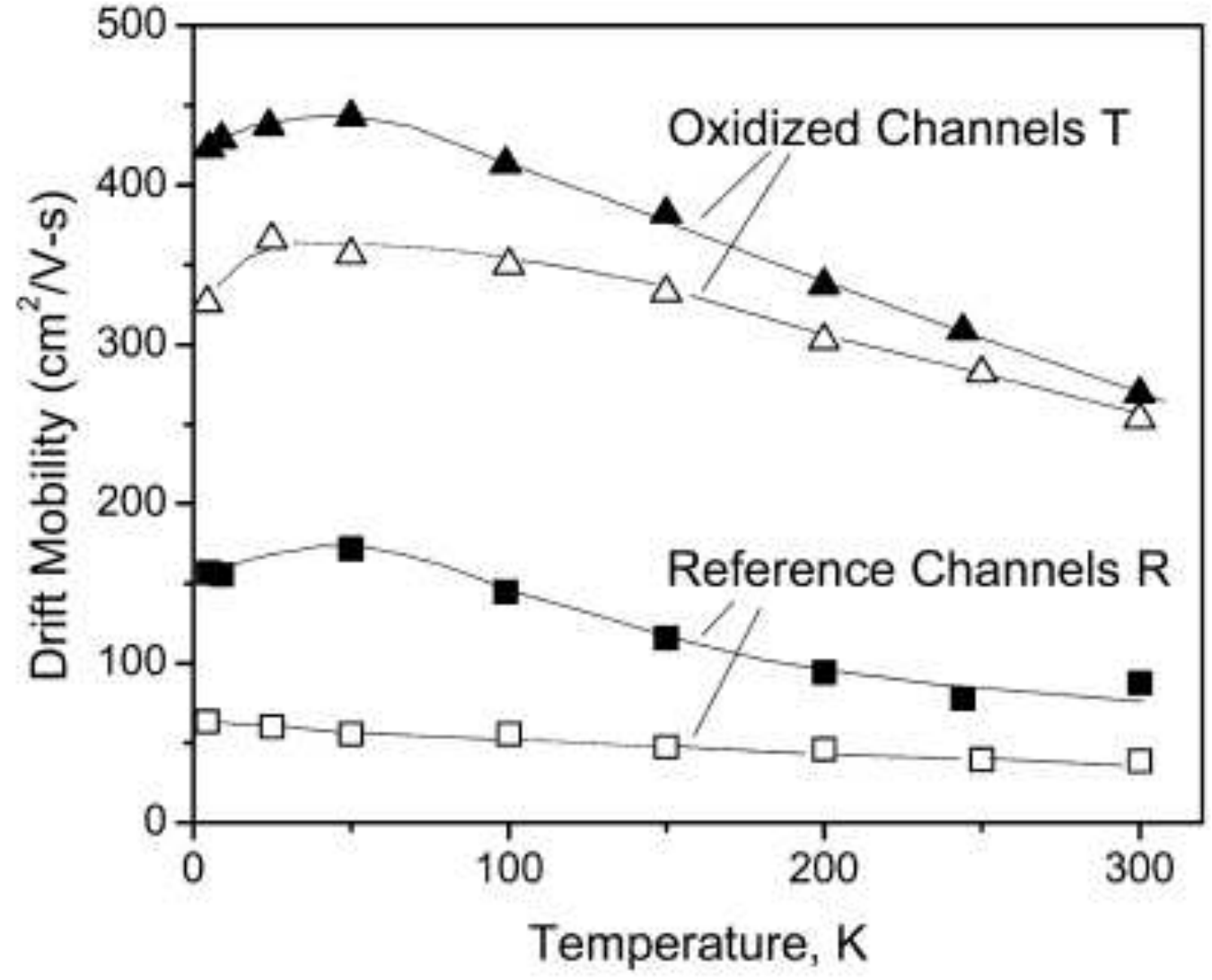

Figure 7 